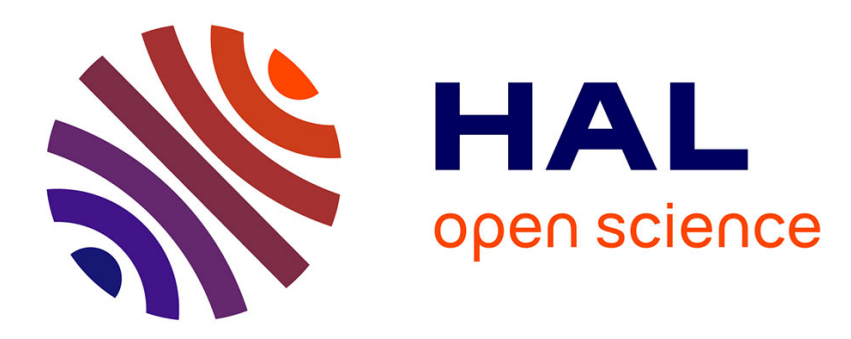

\title{
Un procès d'avortement à Constantinople au 14e siècle
}

\author{
Marie-Hélène Congourdeau
}

\section{To cite this version:}

Marie-Hélène Congourdeau. Un procès d'avortement à Constantinople au 14e siècle. Revue des études byzantines, 1982, 40, pp.103-115. halshs-00683298

\section{HAL Id: halshs-00683298 \\ https://shs.hal.science/halshs-00683298}

Submitted on 28 Mar 2012

HAL is a multi-disciplinary open access archive for the deposit and dissemination of scientific research documents, whether they are published or not. The documents may come from teaching and research institutions in France or abroad, or from public or private research centers.
L'archive ouverte pluridisciplinaire HAL, est destinée au dépôt et à la diffusion de documents scientifiques de niveau recherche, publiés ou non, émanant des établissements d'enseignement et de recherche français ou étrangers, des laboratoires publics ou privés. 


\section{Madame Marie-Hélène Congourdeau}

\section{Un procès d'avortement à Constantinople au 14e siècle}

In: Revue des études byzantines, tome 40, 1982. pp. 103-115.

\section{Résumé}

REB 401982 France p. 103-115

Marie-Hélène Congourdeau, Un procès d'avortement à Constantinople au 14e siècle. - En 1370, à Constantinople, au cours d'un procès de sorcellerie devant le synode présidé par le patriarche Philothée Kokkinos, le hiéromoine Joasaph, du monastère de l'Hodigi- tria, est accusé d'avoir fait avorter une moniale pour cacher leurs relations coupables. II est déposé de son sacerdoce. L'auteur donne la traduction du texte (édité au tome I de Miklosich-Müller), le replace dans le contexte constantinopolitain du 14e siècle et le situe par rapport à l'évolution de la législation impériale et canonique sur l'avortement. Ce procès est ainsi l'occasion d'étudier comment l'autorité ecclésiastique se comportait devant ce problème.

Citer ce document / Cite this document :

Congourdeau Marie-Hélène. Un procès d'avortement à Constantinople au 14e siècle. In: Revue des études byzantines, tome 40, 1982. pp. 103-115.

http://www.persee.fr/web/revues/home/prescript/article/rebyz_0766-5598_1982_num_40_1_2132 


\title{
UN PROCÈS D'AVORTEMENT À CONSTANTINOPLE AU $14^{\mathrm{E}}$ SIÈCLE
}

\author{
Marie-Hélène CONGOURDEAU
}

En 1370, à Constantinople, les retombées d'un procès de sorcellerie mettent au jour une pitoyable histoire d'avortement où sont impliqués un moine et une moniale. Cette affaire peut être l'occasion de voir comment un tribunal ecclésiastique (le synode présidé par le patriarche Philothée Kokkinos) applique l'arsenal juridique et canonique byzantin sur l'avortement. L'étude de ce procès n'a pas pour objectif de rechercher l'attitude officielle de Byzance par rapport à l'avortement (on se doute qu'elle était contre), mais comment, dans la pratique, l'autorité (en l'occurrence religieuse) se comportait à son égard. C'est une pièce apportée au dossier de l'histoire des attitudes devant la vie et la mort, où les études byzantines sont pour le moment moins richement représentées que l'Antiquité ou l'Occident médiéval et moderne ${ }^{1}$.

Le texte, présenté dans les Regestes du Patriarcat de Constantinople ${ }^{2}$, est édité au tome 1 de Miklosich-Müller ${ }^{3}$. Nous nous en tenons au cas d'avortement ${ }^{4}$. Cette affaire, qui met en cause un hiéromoine du monastère de la Théotokos Hodigitria et une moniale du monastère Saint-André-in-

1. Cf. Annales de Démographie Historique (cité par la suite sous le sigle $A D H$ ), 1973 ; Dossier sur l'avortement, L'histoire, $\mathrm{n}^{\circ} 16$, octobre 1979.

2. J. Darrouzès, Regestes, $\mathrm{n}^{\circ 5}$ 2572-2575.

3. MM, I, p. 541-550.

4. Regestes, $n^{\circ} 2574$. 
Krisi, a été évoquée à plusieurs reprises ${ }^{5}$; elle l'a toujours été à propos de la sorcellerie ou de la fornication monastique, mais jamais, à notre connaissance, à propos de l'avortement.

J. Darrouzès a fait l'analyse diplomatique de ce texte ${ }^{6}:$ il s'agit du procès-verbal, inscrit sur le registre des actes synodaux, du grand procès de sorcellerie qui eut lieu à Constantinople dans les années 1370-1371 et dans lequel furent impliqués des personnages importants de la hiérarchie ecclésiastique constantinopolitaine. Ce procès-verbal résume plusieurs procédures et séances judiciaires.

Rappelons l'affaire : le procès, dont la conclusion eut lieu « le 12 mai, indiction 8, le patriarche présidant aux catéchuménies du côté droit de la Grande Eglise (c'est-à-dire dans les tribunes de Sainte-Sophie), siégeant avec lui les (métropolites) de Nicée, Brysis, Chalcédoine, Sozopolis et l'évêque élu de Barna " ${ }^{7}$, est l'aboutissement d'une grande enquête ordonnée par le patriarche Philothée Kokkinos sur des faits de sorcellerie. Quatre accusés principaux sont en cause : le faux moine Phoudoulès, accusé d'avoir entraîné des femmes à s'adonner à la sorcellerie, accuse à son tour le médecin Syropoulos de lui avoir fourni ses livres de magie ; ce dernier dit les tenir d'un certain Gabriélopoulos, chez qui une perquisition permet de découvrir effectivement des livres de magie compilés par Démétrios Chlôros, protonotaire de la Grande Eglise, déjà condamné autrefois comme disciple de Barlaam et Akindynos et acquitté sous condition. Ledit Chlôros, récidiviste et parjure, est déposé de son sacerdoce et enfermé au couvent de la Péribleptos. Les autres accusés sont bannis de l'empire ; étant laïcs, ils subissent une peine temporelle.

C'est alors que l'affaire rebondit, Syropoulos s'avisant de dénoncer des "clients" de son activité de sorcier ; comparaissent donc trois nouveaux accusés : le papas Iôannès Paradeisos, qui a eu recours aux services de Syropoulos pour échapper par des incantations à une condamnation canonique, le papas Iôasaph et le papas Iakob, coupable d'avoir accepté de l'argent pour faire des incantations. Ces trois prêtres sont déposés de leur sacerdoce.

C'est le papas Iôasaph qui nous intéresse. Nous donnons la traduction du passage qui le concerne.

5. Cf. R. JANIN, Eglises et monastères (de Constantinople) ${ }^{2}$, Paris 1969, p. 28 s., 199 s.

6. J. DARrouzès, Le Registre synodal du Patriarcat byzantin au XIV $V^{\mathbf{e}}$ siècle, Paris 1971, p. 208.

7. Regestes, $\mathrm{n}^{\circ} 2572$. 
« Un moine du vénérable monastère de Notre Dame Très Pure et Théotokos Hodigitria, le papas Iôasaph, commit l'impureté avec une moniale du vénérable monastère du saint et glorieux mégalomartyr André-in-Krisi ; voyant qu'elle avait conçu et portait un enfant en son sein, et voulant que le mal demeurât caché et ne fût jamais découvert, comme s'il pouvait demeurer caché même aux yeux de Dieu, il trouva une machination grâce à laquelle il pensait que son forfait ne deviendrait pas visible et manifeste. C'était une pensée diabolique, une invention et une œuvre de celui qui a provoqué aussi la transgression originelle. En effet, cet homme vint trouver Syropoulos et, lui ayant donné un manteau, un vase en verre d'Alexandrie et cinq hyperpères, obtint de lui un produit qu'il rapporta à la moniale, afin que l'ayant bu elle détruisît l'embryon. Elle, l'ayant fait, expulsa ce qu'elle portait en son sein, et l'on ne sut ni les conséquences de la faute, ni cet audacieux forfait. C'est pourquoi jusqu'à ce jour le papas Iôasaph accomplissait les actes de son sacerdoce à visage découvert.

Le papas Iôasaph se présenta donc et eut devant les yeux Syropoulos qui l'accusait et voulait apporter ce qu'il lui avait donné. Ayant entrepris de le contredire, il n'en eut pas la force et, tombant à terre, il s'écria : " $\mathrm{J}$ 'ai péché, très saints et sacrés maîtres ; à présent je reconnais quelle grande perdition $\mathrm{j}$ 'ai procurée à mon âme misérable. Car si, me tenant devant le présent tribunal, je crains et tremble ainsi, et que je perds presque le sens même, qu'éprouverai-je lorsque je me tiendrai devant le juge incorruptible pour rendre compte de mes actes ? C'est pour cela que je confesse la vérité de ce qu'a dit Syropoulos : j'ai commis l'impureté avec cette moniale et je suis devenu le meurtrier de cet embryon ; et maintenant je viens et $j$ 'avoue le mal que $j$ 'ai fait ; et tout ce qui est conforme aux saints et sacrés canons et qui vous semble bon, faites-le"'.

Le papas Iôasaph ayant ainsi rapporté ces faits, le saint synode et notre très saint maître le patriarche œcuménique décrétèrent que celui-ci serait dépouillé de son seul sacerdoce et que cette seule peine serait suffisante pour tous ses forfaits, étant donné que ce n'est pas d'un honneur médiocre qu'il était privé, mais le misérable fut déchu de la dignité sacerdotale, retranché du chœur des prêtres et rejeté loin de la gloire et du grand honneur qu'il possédait. »

Notre étude comportera trois étapes : l'accusation de Syropoulos qui nous indique les faits, la séance au tribunal et la sentence.

\section{LES FAITS}

Le papas Iôasaph est sous le coup de trois accusations : sorcellerie (pour avoir eu recours à Syropoulos), fornication (d'un hiéromoine avec une moniale, circonstance aggravante qui d'après les canons apparente le délit à un adultère) et avortement. Notons au passage que nous ne trouvons nulle part trace de la moniale ; tout ce que nous savons d'elle est qu'elle professait au monastère Saint-André-in-Krisi, situé au sud-ouest de Cons- 
tantinople, loin de l'Hodigitria, monastère de Iôasaph, qui se trouve au sud-est, près du Bosphore. Ce silence est d'autant plus insolite que la législation condamne surtout la femme qui avorte, éventuellement la faiseuse d'anges ou le fabricant de potions, jamais le père : c'est pourtant lui que nous avons ici.

Laissons provisoirement de côté les autres chefs d'accusation pour retenir celui d'avortement. Le moyen employé (un breuvage) est le plus fréquemment cité dans les textes officiels. Nous avons peu de renseignements sur la composition de ces drogues, qui devaient être à base d'herbes particulièrement toxiques puisqu'elles étaient souvent fatales à celles qui les absorbaient. Les procédés mécaniques (pessaires ${ }^{8}$, poids pour comprimer le ventre ${ }^{9}$ ou autres) paraissent moins employés ou plus clandestins.

Les textes évoquent presque toujours, comme motif de l'avortement, une conception illégitime ; elle l'est particulièrement ici. Le cas, plutôt scabreux, n'est pas aussi extraordinaire qu'il apparaît à nos consciences modernes. J.-L. Flandrin ${ }^{10}$, parlant non d'avortement mais d'infanticide, cite le Pénitentiel occidental de Finnian qui punit lourdement le clerc fornicateur et plus lourdement celui qui, ayant conçu un enfant, le tue pour éviter le scandale. L'Occident n'est pas mieux loti sur ce point que Byzance.

A Byzance même, que se passait-il au moment où le papas Iôasaph cherchait à faire disparaître le fruit de ses amours illicites ?

Constantinople au $14^{\mathrm{e}}$ siècle. - Un texte de Joseph Bryennios, édité par L. Oeconomos ${ }^{11}$, nous donne une peinture peu flatteuse des mœurs byzantines à cette époque, responsables selon l'auteur des malheurs du temps. On y retrouve, entre autres, les chefs d'accusation imputés au papas Iôasaph. La sorcellerie : "Nous nous adressons journellement aux magiciens, aux devins, aux tziganes, aux sorciers; pour toute maladie nous avons recours à la sorcellerie... "; la fornication : "C'est sans honte que les moines, en dépit de leur vœu de chasteté, cohabitent avec des nonnes... »; exercice sacrilège du sacerdoce : "Bien des membres du clergé, après s'être comportés d'une manière dissolue, s'approchent de la Sainte Table

8. Hippocrate, Des maladies des femmes, I, 78, cité par R. Etienne, La conscience médicale antique et la vie des enfants, $A D H, 1973$.

9. Zonaras : Rhallès-Potlès, III, p. 63-64.

10. J.-L. Flandrin, L'attitude à l'égard du petit enfant et les conduites sexuelles dans la civilisation occidentale, $A D H, 1973$, p. $160 \mathrm{~s}$.

11. L. CEConomos, L'état intellectuel et moral des Byzantins vers le milieu du $14^{*}$ siècle d'après une page de Joseph Bryennios, Mélanges Charles Diehl, I, Paris 1930, p. 225. 
et officient. O épargne-nous, Seigneur. C'est surtout ce dernier péché plus que tous les autres qui nous attire la haine de Dieu et nous expose à mille maux. "

Au début de ce $14^{e}$ siècle, le patriarche Athanase $I^{\text {er }}$ multiplie lui aussi les mises en garde : «Ce ne sont partout que pratiques de magie ou de divination, adultères, fornication, sodomie, homosexualité, vols, meurtres, usure, ivrognerie, blasphèmes et autres abominations " ${ }^{12}$. "Ils (les fidèles) devront fuir les femmes qui donnent ou prennent des produits en vue de provoquer l'avortement $»^{13}$. «Excommuniés (sont) ceux qui s'adonnent à des pratiques bizarres de sorcellerie (...), ceux qui tiennent des maisons de tolérance ainsi que les femmes qui distribuent ou acceptent des médicaments pour avorter $»^{14}$. «Prescription 18 : En cas de maladie (...), ne recourir ni aux mages ni aux devins (...). Prescription 19 : Traiter comme une criminelle la femme qui prend des produits pour éliminer l'enfant ou le fœtus ainsi que celle qui expose ses petits " ${ }^{15}$. «On ne recourra ni aux mages ni aux devins ni aux sorciers... On avertira les femmes qui donnent ou font connaître des produits pour avorter ainsi que celles qui jettent leurs enfants qu'elles encourent les mêmes peines et les mêmes censures que les criminelles ${ }^{16}$.

En six ans de patriarcat (1303-1309), Athanase $I^{\text {er }}$ mentionne quatre fois l'avortement. Tout en faisant la part de la prolixité grecque, on ne peut manquer d'être frappé par le tableau qui se dégage de ces textes.

Un coup d'œil sur les procès de prêtres ou de moines, tels qu'on peut les trouver dans les fascicules V et VI des Regestes du Patriarcat de Constantinople, couvrant les années 1310-1410, confirme par des exemples et non plus des discours que les moines byzantins du $14^{\mathrm{e}}$ siècle n'étaient pas tous aussi « angéliques » que leurs confrères hésychastes l'eussent souhaité.

Laissons de côté quelques cas pittoresques d'ivrognerie ${ }^{17}$. Il nous reste des moines condamnés pour avoir eu recours à des mages ou devins ${ }^{18}$ ou pour avoir eux-mêmes pratiqué la magie ${ }^{19}$. Pendant la même période,

12. Nous citons les résumés de J. Darrouzès, Regestes, $\mathrm{n}^{\circ} 1589$.

13. Lettre patriarcale aux évêques, prêtres et higoumènes ; Regestes, $\mathrm{n}^{\circ} 1749$.

14. Mandement à tous les métropolites du Patriarcat ; Regestes, $\mathrm{n}^{\circ} 1747$.

15. Regestes, $\mathrm{n}^{\circ} 1762$.

16. Regestes, $\mathrm{n}^{\circ} 1779$.

17. Regestes, $\mathrm{n}^{\text {os }} 2638,2877$.

18. Regestes, $\mathrm{n}^{\text {os }} 2334,2489$.

19. Regestes, $\mathrm{n}^{\circ} 2770$ : il s'agit d'ailleurs de l'incorrigible Phoudoulès qui en 1384 , à l'approche de la mort, avoue n'avoir pas abandonné ses activités de sorcier. 
six moines ou hiéromoines au moins subissent des condamnations pour relations coupables ou suspectes avec des femmes de mauvaise vie, voire des moniales telle cette Thiniatissa qui «résidait dans sa propre maison dont elle avait fait un mauvais lieu comme entremetteuse $»^{20}$. Parmi eux, un papas Iôasaph de l'Hodigitria qui, en 1355 (quinze ans avant notre affaire), était suspens de son sacerdoce pour avoir été pris en flagrant délit de fornication dans sa cellule. Rien n'indique s'il s'agit du même Iôasaph.

Malgré les précautions prises par les patriarches ${ }^{21}$ pour empêcher toute communication entre les moines et les femmes, moniales ou non, une certaine frange de la population monastique de Constantinople, dans laquelle nous inclurons le papas Iôasaph, n'avait pas une conduite conforme à son habit. C'est dans ce contexte qu'il nous faut juger cette affaire.

\section{LE PROCÈS}

Le papas Iôasaph, en tant que moine et prêtre de Constantinople, relève de la juridiction patriarcale. Le canon 20 du concile de Carthage spécifie qu'en matière criminelle, un prêtre doit être jugé par six évêques dont son évêque propre. C'est le cas ici, puisque le synode comprend - outre le patriarche, évêque propre de l'accusé - cinq autres évêques : ceux de Nicée, Brysis, Chalcédoine, Sozopolis et Barna.

Ce privilège n'allait pas sans contestation puisqu'en 1396, lors d'un procès en diffamation où se trouvaient impliqués l'empereur Manuel II et le patriarche Antoine, l'empereur imposa au synode la présence de juges civils, malgré un vote du synode qui " (récusait) les juges civils et (déniait) à l'empereur le droit de s'immiscer dans les procès criminels ecclésiastiques ${ }^{22}$. Tel n'est pas le cas ici et le papas Iôasaph, jugé par ses pairs, recevra une pénitence canonique accordée à son état.

Le déroulement du procès est sans surprise. L'accusé a été l'objet d'une dénonciation. Le cas est banal : maint texte juridique évoque le statut du dénonciateur. Comme il n'y a qu'un seul témoin, l'aveu du prêtre est important pour ses juges. Il l'est encore plus pour l'historien, car la forme de cet aveu ("Je suis devenu le meurtrier de cet embryon ") révèle un consensus entre l'accusé et la justice : le caractère criminel de l'acte n'est pas contesté par son auteur. Certes, il s'agit d'un prêtre : accusé et juges

20. Regestes, $\mathrm{n}^{\circ} 2339$; cf. $\mathrm{n}^{\circ \mathrm{s}} 2385,2450,2816,2887,2970$.

21. Cf. les instructions d'Athanase $I^{\text {er }}:$ Regestes, $n^{\text {os }} 1595$ et 1776.

22. Regestes, $\mathrm{n}^{\circ} 3025$. 
appartiennent à la même société. Mais le personnage est assez fruste pour représenter aussi un bon témoin de l'autre société, celle dont il est issu : le peuple de Constantinople. Son aveu peut être considéré comme un indice valable de l'opinion populaire.

\section{LA SENTENCE}

A la fin de notre texte, les membres du synode semblent vouloir justifier leur sentence comme s'ils craignaient qu'elle ne fût jugée trop clémente. Tâchons d'apprécier cette sentence en la situant dans le contexte de la législation byzantine.

1. LA LÉGisLation SUR L'AVORTEMENT. Résumons brièvement les prescriptions impériales et canoniques en matière d'avortement.

a. La législation impériale. Celle-ci est en grande partie l'héritière du droit romain tel qu'il est compilé dans le Digeste de Justinien. Elle concerne principalement la femme qui avorte : elle est condamnée à l'exil temporaire, que son acte soit dicté par la haine d'un mari qui l'a répudiée ${ }^{23}$ ou par tout autre motif ${ }^{24}$, excepté le cas où des héritiers en second l'auraient payée pour faire disparaître l'héritier principal ${ }^{25}$. Ce cas, tiré d'une anecdote que Cicéron rapporte dans son Pro Cluentio ${ }^{26}$, aura une riche destinée puisqu'on le retrouve dans la plupart des textes byzantins ${ }^{27}$. On verra dans la fortune de ce cas d'école un exemple du caractère répétitif de la législation byzantine plus qu'un témoignage sur une pratique sans doute assez rare. Le second élément que l'on trouve dans le Digeste concerne les fabricants de drogues, parmi lesquelles les abortifs, qui sont condamnés, s'ils sont de haut rang, à l'exil avec confiscation des biens; s'ils sont de basse extraction, au travail forcé dans les mines. Si la femme succombe, c'est la peine de mort $^{28}$.

23. Digeste, 48, 19, 39, repris dans le Procheiron auctum, 39, 186 et les Basiliques, 60, 51,35 .

24. Digeste, 47, 11, 4 ; 48, 8, 8 et Procheiron auctum, 39, 185.

25. Digeste, $48,19,39$.

26. Cicéron, Pro Cluentio, 11, 32.

27. Ecloga ad Procheiron mutata, 35, 1 ; Procheiron auctum, 39, 186 ; Epanagoge, 40, 7 ; Basiliques, 60, 51, 35.

28. Digeste, $48,19,38,5$. 
Reprendront ces dispositions l'Ecloga ad Procheiron mutata ${ }^{29}$, le Procheiron auctum $^{30}$, les Basiliques ${ }^{31}$ et l'Epitomè ${ }^{32}$. A l'exil temporaire, l'Ecloga ajoute, au $8^{\mathrm{e}}$ siècle, la flagellation pour la femme qui avorte ${ }^{33}$. Cette loi restera en vigueur, sans pour autant annuler les précédentes ${ }^{34}$. Seul mobile évoqué par cette loi : la fornication et le désir d'en effacer toute trace. Le Procheiron auctum et l'Epitomè ${ }^{35}$ indiquent que quiconque donne ou reçoit un abortif est un homicide, sans précision de peine. Enfin Léon VI, dans ses Novelles, donne au mari dont la femme a avorté licence de s'en séparer ${ }^{36}$.

On le voit, la législation traite principalement de la femme qui avorte, de la faiseuse d'anges et des fabricants de drogues (accessoirement abortives). Une particularité de l'Epitomè ${ }^{37}$ : celui qui fait avorter une femme

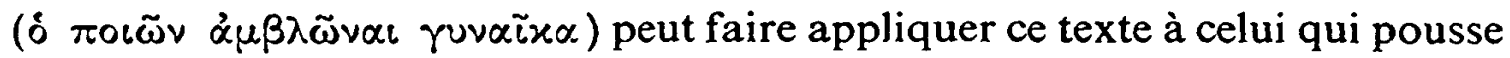
la femme à avorter (ici le père), bien qu'il semble plutôt désigner celui qui "pratique » l'avortement.

Voilà quelle était la législation appliquée dans le siècle, qu'il serait intéressant à l'occasion de confronter à un procès réel. Le coupable étant ici un prêtre, relevant comme tel du tribunal ecclésiastique, voyons à présent la législation canonique.

b. Le droit canon. Là encore, les textes ne parlent que de femmes : celle qui avorte et celle qui fournit la drogue : on peut y voir un indice de ce que l'avortement est avant tout en ce temps une affaire de femmes, comme tout ce qui touche à la grossesse et à la naissance. Cependant les termes sont suffisamment imprécis (plus que le «fabricant de drogues» impérial) pour concerner au besoin celui qui donne la potion.

Une « ancienne loi » non identifiée ${ }^{38}$ mais citée par le concilc d'Ancyre

29. Digeste, 35,2 .

30. $39,183,188$.

31. $60,51,34,5$.

32. $45,20$.

33. 17,36 .

34. Elle est rappelée dans l'Ecloga aucta $(17,19 ; 27)$, l'Ecloga ad Procheiron mutata $(19,17 ; 21,18)$ [le bannissement étant dans ces deux textes remplacé par la confiscation], le Procheiron auctum $(39,182)$, l'Epanagogè $(40,65)$, l'Epanagogè aucta $(52,64)$, les Basiliques $(60,22,4)$.

35. Procheiron auctum, 39, 219 ; Epitome, 45, 7.

36. Novelle 31.

37. Epitomè, $45,7$.

38. Un canon occidental du concile d'Elvire en Espagne, en 300, exclut de la communion la femme qui avorte, jusqu'à son lit de mort. Les termes de ce canon seraient-ils parvenus jusqu'aux Pères d'Ancyre ou faut-il chercher en Orient une autre "ancienne loi » aux termes identiques? 
de 314 prévoit pour ces femmes la privation des sacrements jusqu'au lit de mort ; le canon 21 du concile d'Ancyre ${ }^{39}$ adoucit cette loi et limite la pénitence à dix ans : la pénitente devra pendant ces dix ans suivre les degrés fixés pour la pénitence publique. Les canons 2 et 8 de Basile de Césarée ${ }^{40}$, qui serviront de référence à tous les textes postérieurs, imposent la peine du meurtre à celle qui donne comme à celle qui reçoit l'abortif, quel que soit l'âge de l'embryon (allusion à une abondante querelle qui remonte à l'Antiquité et traverse toute la civilisation gréco-latine jusqu'à nos jours, sur le point de savoir à partir de quel âge l'embryon est un être humain); outre le respect de l'embryon, Jes risques courus par la femme sont un des mobiles qui animent saint Basile (indice, avec l'aggravation de la peine des avorteurs en cas de mort de la femme, de l'importante mortalité féminine due à l'avortement).

On notera avec intérêt la façon dont est conçue cette peine du meurtre : selon le concile d'Ancyre (canon 22), le meurtre volontaire est taxé de la privation des sacrements jusqu'au lit de mort ; cependant, ce même concile ne donne que dix ans de pénitence à la femme qui avorte, et Basile confirme cette durée : or, c'est celle qu'il attribue à la peine du meurtre involontaire (canons 11 et 57) : est-ce, comme le suppose Balsamon ${ }^{41}$, parce qu'il lui accorde des circonstances atténuantes ou que le meurtre de l'embryon n'est pas aussi grave que le meurtre d'un être né, ou est-ce parce que n'est alors puni que le risque de tuer la femme, dont la mort ne peut être qu'involontaire ? Cette question méritera une étude particulière qui ne peut tenir dans le cadre de cet article.

Le canon 91 du concile in Trullo en 692 n'ajoute rien, se contentant de prévoir la peine du meurtre pour celles qui donnent ou reçoivent un poison abortif. Les textes suivants (Nomokanon de Photius au $9^{e}$ siècle, Syntagma de Matthieu Blastarès au $14^{e}$ siècle) ne sont que des compilations des canons d'Ancyre, de Basile et du concile in Trullo, auxquels ils adjoignent quelques lois impériales : celles du Digeste (Photius et Blastarès) et la Novelle de Léon VI (Blastarès).

Les textes de jurisprudence canonique, en particulier les Pénitentiels, nous renseignent un peu sur la façon dont étaient appliqués ces canons : le plus instructif est le Kanonikon dit de Jean le Jeûneur, daté du $10^{c}$ siècle ; en effet, s'il rappelle les canons prévoyant la peine du meurtre, il est d'avis (selon sa pratique coutumière) de ramener la pénitence à cinq ou même

39. Rhallès-Potlès, III, p. 63.

40. Lettre 188 à Amphiloque sur les canons, paragraphes 2 et 8 .

41. Balsamon : Rhallès-Potlès, IV, p. $97 \mathrm{~s}$. 
trois ans ${ }^{42}$ : ce faisant, il ne fait qu'appliquer ses propres prescriptions sur le meurtre, puisqu'il prévoit cinq ans pour le meurtre volontaire et trois ans pour le meurtre involontaire.

$\mathrm{Au}$ vu de ce dossier, examinons à présent le cas du papas Iôasaph. N'oublions pas que nous avons affaire à un prêtre.

2. LA LÉGislation SUR LE PRÊTRE. Elle est très simple. Depuis les canons des Apôtres ( $3^{e}$ siècle), la peine pour le prêtre meurtrier comme pour le prêtre fornicateur est la déposition. Basile de Césarée précise d'ailleurs que c'est la peine qui s'applique aux prêtres coupables d'un péché mortel, quel qu'il soit; les textes postérieurs ne feront que reprendre cette prescription.

C'est donc sans surprise que nous la voyons appliquée au papas Iôasaph : qu'il soit jugé comme clerc fornicateur ou qu'on lui applique, conformément à la législation sur l'avortement, la peine du meurtre, il est passible de déposition. Ce qui étonne, c'est que les juges synodaux semblent vouloir se défendre de porter un jugement trop clément. Certes, l'accusé s'est rendu coupable d'un certain nombre de péchés mortels : fornication ecclésiastique (qui prend la forme d'un double adultère), séduction d'une moniale, recours à la sorcellerie, sacrilège. Mais outre qu'il s'agit là d'un enchaînement de fautes, la décision du synode est conforme aux canons 3, 32 et 51 de Basile : "Ces canons ont exposé sans donner de précision ce qui concerne les clercs et ils ont ordonné qu'une seule peine fût infligée à ceux qui sont tombés : l'exclusion de leur ministère " (canon 51). Aucune pénitence supplémentaire ne sera infligée à celui qui reçoit la pénitence suprême : la déposition.

La sentence portée contre le papas Iôasaph étant reconnue conforme aux canons, il reste à la comparer avec la jurisprudence synodale.

Jurisprudence synodale. Si nous étudions, dans les fascicules V et VI des Regestes, les sentences portées contre des clercs au $14^{\mathrm{e}}$ siècle, nous trouvons essentiellement deux peines :

La «suspense » (privation temporaire du ministère) réprime des délits d'ivrognerie ${ }^{43}$, de calomnies ${ }^{44}$, de relations douteuses avec une femme ${ }^{45}$,

42. Kanonikon : Rhallès-Potlès, IV, p. 443. Cf. Didaskalia monazousôn, 10 : le texte est particulièrement bien adapté à notre affaire, puisque l'auteur ne juge pas extraordinaire d'avoir à prévoir une peine pour les moniales qui avortent.

43. $\mathrm{N}^{\circ} 2877$.

44. No 2902.

45. Nos $2816,2970$. 
de violences et injures ${ }^{46}$, d'exercice public d'une profession lucrative ${ }^{47}$.

La "déposition" concerne des complots politiques ${ }^{48}$, des infractions aux lois matrimoniales ${ }^{49}$, des sacrilèges ${ }^{50}$, la magie ${ }^{51}$ ou la fornication notoire ${ }^{52}$ : toutes fautes incompatibles avec l'exercice du sacerdoce. Nous relèverons enfin le cas d'un hiéromoine de Pharos qui, en 1372, fut accusé d'avoir livré aux Turcs des chrétiens réfugiés : «Les membres du synode et le patriarche (décidèrent) que les laïcs (recevraient) les sanctions canoniques du meurtrier et que le hiéromoine (serait) déposé ${ }^{53}$. La déposition correspond bien pour le prêtre à la « peine du meurtre " chez les fidèles.

Si la déposition est la peine la plus forte prononcée contre un prêtre, le synode n'avait pas à justifier sa sentence : son embarras montre donc que l'avortement apparaissait bien aux yeux du peuple comme une faute exceptionnellement grave.

$\mathrm{Au}$ terme de cette étude, qu'avons-nous appris sur l'avortement à Byzance ? Nous retrouvons dans ce procès le trio classique de l'avortement : la mère, l'avorteur, le juge.

La mère est absente. Peut-être a-t-elle succombé à l'absorption du breuvage; mais on peut penser que sa mort aurait été relevée comme circonstance aggravante. Plus sûrement, en tant que femme et moniale, elle n'a pas à comparaître dans un grand procès public : son cas relève de la compétence de son confesseur. Mais son rôle est tenu ici par le moine ; par lui nous connaissons le mobile (cacher une conception illégitime) qui dans cette société doit représenter la majorité des avortements ; c'est presque le seul mobile évoqué par la loi. Nous mesurons aussi l'impact psychologique de l'acte : l'effondrement de Iôasaph, les termes de son aveu montrent qu'il a conscience d'avoir tué. Quelles que soient les discussions d'intellectuels sur l'âge à partir duquel l'embryon est un être humain, on peut dire que pour la majorité des Byzantins l'avortement est la destruction d'une vie humaine.

L'avorteur, c'est Syropoulos, le "fabricant de drogues» réprimé par la loi plus que la "bonne femme» avec ses méthodes empiriques. Lorsque

46. $\mathrm{N}^{\circ} 2851$.

47. No 2857.

48. $\mathrm{N}^{\text {os }} 2927,3025$.

49. $N^{\text {os }} 2863,2480,2531$, etc.

50. Nos $2498,2595,2604$.

51. No 2648.

52. $\mathrm{N}^{\circ} 2385$.

53. $N^{\circ} 2647$. 
l'affaire Iôasaph est évoquée, il est déjà condamné à l'exil dont il serait passible pour cet avortement. C'est un médecin suspect de sorcellerie : nous ne savons pas au juste si Iôasaph a eu recours au médecin ou au sorcier ; si c'est au médecin, cela pose le problème des rapports entre la

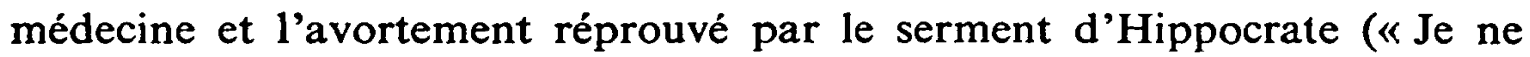
donnerai à aucune femme un pessaire abortif ${ }^{54}$ ). Si c'est au sorcier, ce sont les rapports entre la sorcellerie et la confection de drogues et poisons (pensons à la " sorcière » de nos villages occidentaux et à ses herbes maléfiques). Quoi qu'il en soit, par l'emploi des herbes et potions, les deux sont liés, et c'est le fond du procès de 1370. Par Syropoulos, nous connaissons le prix de la potion qu'il a composée : un manteau, un vase en verre d'Alexandrie et cinq hyperpères. Curieuse association du troc et de la monnaie ; il est vrai que Iôasaph, de par son vœu de pauvreté, ne devait pas être riche en numéraire. Ce prix, qui nous semble élevé pour une simple potion, payait-il le silence du fournisseur, le risque couru par lui ou le déséquilibre entre l'offre et la demande (voir les plaintes de Joseph Bryennios et du patriarche Athanase) ? Nous manquons d'éléments de comparaison.

Les juges, ce sont les évêques du synode avec leur arsenal juridique. Plus que la société, ils représentent la position de l'Eglise. Intraitable sur le principe, elle accorde volontiers les circonstances atténuantes à la femme. On remarquera l'évolution de la législation : de la privation des sacrements jusqu'à la mort, on passe à une pénitence de dix ans dans les canons, cinq ou trois ans dans la pratique. Les canons se préoccupent aussi de la mortalité féminine.

Dans son attitude devant l'avortement, Byzance se distingue à la fois de l'Antiquité païenne et de l'Occident. Pour les sociétćs païennes de l'Antiquité gréco-latine, on peut dire en gros que l'avortement n'était pas couramment considéré comme un meurtre ${ }^{55}$. Nous avons vu qu'à Byzance, au contraire, ce principe n'est pas contesté. D'autre part, dans la position byzantine, l'âge de l'embryon n'entre pas en ligne de compte. Certes, des discussions ont lieu sur la question des fameux «quarante jours " pendant lesquels, à en croire Aristote, l'embryon n'est pas encore "animé » ou

54. Cf. R. ETIENNE, art. cit. (n. 8), p. 27.

55. Ibidem. La question étant encore en suspens, nous renvoyons à : HartmanN, Abortio, RE, $1 / 1$, p. 108 ; HUMBert, abigere partum, Dictionnaire des Antiquités grecque et romaine, $1 / 1$, p. 7-8 ; IDEM, abortio, ibidem, p. 9-10 ; CAILLEMER, ambloséos graphè, ibidem, p. 224-225 ; WASZINk, Abtreibung, Reallexicon für Antike und Christentum, I, p. 55-60 ; DöLGER, Das Lebenrecht des ungeborenen Kindes und die Fruchtabtreibung in der Bewertung der heidnischen und christlichen Antike, Antike und Christentum, IV, 1933, p. 1-61. 
"formé ». La traduction grecque d'Exode 21, 22 contribuait à alimenter la querelle $^{56}$. Mais au-delà des discussions théoriques, l'Eglise et l'opinion populaire s'en tenaient au canon 2 de Basile qui reste la base de la pratique byzantine : « Nous ne faisons pas d'enquête minutieuse pour savoir s'il était formé ou informe ", et cela, semble-t-il, autant par souci de la mère que de l'enfant : " parce que, comme il arrive le plus souvent, les femmes meurent dans de pareilles tentatives ". C'est sans doute l'originalité principale de Byzance par rapport à l'Occident où les Pénitentiels prévoient une peine différente suivant l'âge de l'embryon ${ }^{57}$.

Enfin, l'Eglise byzantine fait preuve d'une «économie » croissante envers la femme : en cela elle diffère de la législation impériale qui, à partir du $8^{e}$ siècle, aggrave la peine de la femme qui avorte en ajoutant la flagellation à l'exil. Peut-être est-ce la rigidité du principe qui, supprimant l'ambiguïté conceptuelle, donne aux praticiens la liberté d'" adapter » la peine à l'accusé et aboutit à cette position originale, à la fois rigoureuse et nuancée, de l'Eglise byzantine face à l'avortement.

56. "Quand deux hommes se battent et arrivent par là à faire accoucher une femme enceinte d'un enfant non complètement formé, le responsable devra payer une amende fixée par le mari. Mais si le fruit était formé, alors il devra donner vie pour vie. "

57. Cf. J.-L. Flandrin, art. cit. (n. 10), p. 156 ; IDEM, L'Eglise et le contrôle des naissances, Paris 1970, p. 43 s.; NOONAN, Contraception, a history of its treatment by the catholic theologians and canonists, Cambridge (Mass.) 1966. 